ESJ Natural/Life/Medical Sciences

\title{
Indices De Minéralisations En Or Dans La Province Panafricaine Du Sud Maradi (Sud Niger)
}

\author{
Dr. Souley Baraou Idi \\ Université d'Agadez, Faculté des Sciences et Techniques, Département de \\ Géologie P.O., Agadez, Niger \\ Prof. Moussa Konaté
}

Université Abdou Moumouni de Niamey, Faculté des Sciences et Techniques, Laboratoire de Géologie : Eaux Souterraines et Géoressources, Niamey, Niger

\section{Dr. Yacouba Ahmed, M.C}

Université Abdou Moumouni de Niamey, Faculté des Sciences et Techniques, Laboratoire de Géologie : Eaux Souterraines et Géoressources, Niamey, Niger

\section{Dr. Abdoulwahid Sani}

Université d'Agadez, Faculté des Sciences et Techniques, Département de Géologie P.O., Agadez, Niger

Doi:10.19044/esj.2021.v17n21p238

Submitted: 12 May 2021

Accepted: 01 June 2021

Published: 30 June 2021
Copyright 2021 Author(s)

Under Creative Commons BY-NC-ND 4.0 OPEN ACCESS

Cite As:

Baraou Idi S., Konaté M., Ahmed Y. \& Sani A. (2021). Indices De Minéralisations En Or Dans La Province Panafricaine Du Sud Maradi (Sud Niger). European Scientific Journal, ESJ, 17(21), 238.

https://doi.org/10.19044/esj.2021.v17n21p238

\section{Résumé}

Le socle du Sud Maradi (Sud Niger) correspond à l'extrémité Nord du bouclier Bénino-Nigérian, appartenant à la zone mobile panafricaine à l’Est du Craton Ouest-Africain. Cette étude apporte une modeste contribution dans la caractérisation géochimique des minéralisations aurifères de cette province métallogénique panafricaine. A cet effet, l'approche méthodologique mise en œuvre associe le dosage géochimique de l'or par absorption atomique (Au Fire Assay/AA) pour les roches saines, et la séparation des pépites d'or par l'utilisation de la table à secousse pour les altérites du socle et les sédiments (placers et paléoplacers). Les analyses géochimiques ont mis en évidence 
l'existence de deux types de minéralisations: primaires et secondaires. Les minéralisations primaires de l'or sont à l'état disséminé (faibles teneurs de 0.005 à $0.017 \mathrm{~g} / \mathrm{t}$ ) mais des teneurs relativement plus élevées (0.006 à 0.017 $\mathrm{g} / \mathrm{t}$ ) ont été enregistrées au voisinage des zones de cisaillement du socle. Tandis que la minéralisation secondaire, mise en évidence dans les altérites du socle, dans les alluvions (placers) et dans les grès du Crétacé (paléoplacers) présentent des teneurs en or plus élevées (5 à $30 \mathrm{~g} / \mathrm{t}$ ), dépassant largement les teneurs des minéralisations primaires observées dans les roches saines du socle. Ce grand écart de teneurs s'explique par un fort lessivage du socle, qui aurait permis une reconcentration de l'or dans les sédiments.

Mots clés: Sud Maradi, Bouclier Bénino-Nigérian, Zone Mobile Panafricaine, Minéralisation En Or

\title{
Occurrences Of Gold Mineralization In South Maradi Pan- African Province (South Niger)
}

\author{
Dr. Souley Baraou Idi
}

Université d'Agadez, Faculté des Sciences et Techniques, Département de Géologie P.O., Agadez, Niger

\section{Prof. Moussa Konaté}

Université Abdou Moumouni de Niamey, Faculté des Sciences et Techniques, Laboratoire de Géologie : Eaux Souterraines et Géoressources, Niamey, Niger

Dr. Yacouba Ahmed, M.C

Université Abdou Moumouni de Niamey, Faculté des Sciences et

Techniques, Laboratoire de Géologie : Eaux Souterraines et Géoressources, Niamey, Niger

\section{Dr. Abdoulwahid Sani}

Université d'Agadez, Faculté des Sciences et Techniques, Département de Géologie P.O., Agadez, Niger

\section{Abstract}

The South Maradi (South Niger) basement corresponds to the northeastern part of the Benin-Nigerian Shield, belonging to the Pan-African mobile zone, which is located to the east of the West African Craton. This study brings a modest contribution to the geochemical characterization of gold mineralization in this Pan-African metallogenic province. For this purpose, the methodological approach implemented combines the geochemical determination of gold contents by atomic absorption (Au Fire Assay/AA) for basement rocks and physical separation of gold specks by using the vibrating 
table process for basement alterites and sediments (placers and paleoplacers). The obtained results showed two types of gold mineralization: primary and secondary. Primary gold mineralization is in a disseminated state (low contents of 0.005 to $0.017 \mathrm{~g} / \mathrm{t}$ ) but relatively the higher values ( 0.006 to 0.017 $\mathrm{g} / \mathrm{t}$ ) were recorded near the basement shear zones. While the secondary gold mineralization, highlighted in basement alterites, alluvium (placers) and Cretaceous sandstones (paleoplacers) present higher gold contents (5 to $30 \mathrm{~g}$ / t) than primary gold contents observed in basement rocks. This large difference in values can be explained by a strong alteration of the basement, which would have allowed a reconcentration of the gold in the sediments.

Keywords: South Maradi, Benin-Nigerian Shield, Pan-African Mobile Zone, Gold Mineralization

\section{Introduction}

Des gisements d'or ont été décrits dans plusieurs provinces panafricaines d'Afrique de l'Ouest (Goh, 2016), notamment dans le bouclier Bénino-Nigérian qui représente une importante province métallogénique (Samuel and Fakorede, 1988 ; Garba, 2000 ; Talaat et al., 2010 ; Abubakar, 2012). La zone Sud Maradi (latitudes $\mathrm{N} 13^{\circ}$ et $13^{\circ} 20^{\prime}$ et longitudes E $6^{\circ} 50^{\prime}$ et $\left.7^{\circ} 35^{\prime} 00^{\prime \prime}\right)$, objet de cette étude, représente l'extrémité nord de cette province métallogénique, appartenant à la zone mobile panafricaine située à l’Est du Craton Ouest-Africain (Mignon, 1970).

Les premières études de prospection minière dans le Sud Maradi ont été réalisées par Mignon (1970). A ces travaux s'ajoutent des études de prospection géophysique aéroportée effectués par le PRDSM (2005). Toutes ces études ont mis en évidence des indices d'or dans les alluvions charriées par les deux principaux cours d'eaux: le Goulbi N'Maraka et le Goulbi $N$ 'Nielwa. Depuis ces travaux de reconnaissance, aucune étude détaillée sur la caractérisation géochimique des minéralisations aurifères n’a été entreprise.

La présente étude vise à améliorer les connaissances sur les minéralisations aurifères de cette portion de la zone mobile panafricaine. Il s'agit spécifiquement de déterminer les teneurs en or dans les formations de socle et dans les sédiments puis d'établir un modèle préliminaire de distribution spatiale des indices aurifères dans la zone étudiée.

\section{Contexte géologique de la zone d'étude}

La zone d'étude (Sud Niger) correspond à la bordure Nord du bouclier Bénino-Nigérian, située à l'Est du Craton Ouest Africain et à l'Ouest du Craton congolais (Figure 1). Les travaux géologiques réalisés dans la province contiguë du Nord Nigéria ont démontré que les déformations se sont produites dans un contexte d'orogenèse polyphasée qui a affecté le bouclier 
Bénino-Nigérian (Turner, 1983 ; Fitches et al., 1985 ; Grant, 1970; Ajibade et al., 1987; Dada 1998). Plusieurs auteurs s'accordent sur le fait que le socle polycyclique du Nord-Ouest du Nigéria correspond à une ancienne croûte comprenant des panneaux archéens (2700 \pm 200 Ma) et éburnéens (2000 \pm 200 Ma, Grant, 1970; Ajibade et al., 1987; Dada 1998). Le bouclier BéninoNigérian est un vaste ensemble, affecté par l'évènement thermo-tectonique panafricain pendant la période allant du Néoprotérozoïque au Cambrien (750 Ma -500 Ma) - Kennedy, 1964 ; Villeneuve \& Cornée, 1994 ; Stern, 1994 ; Trompette, 1997 ; Castaing et al., 1993 ; Abdelsalam et al., 2002 ; Liégeois et al., 2003 ; Caby, 2003-. L’orogenèse panafricaine (600 $\pm 150 \mathrm{Ma})$ est la dernière réactivation qui a affecté toute la région (Turner, 1983; Fitches et al., 1985; Wright et al., 1985). Ce dernier évènement thermo-tectonique serait associé au fonctionnement de structures cisaillantes panafricaines vers $505 \pm$ 10 Ma K-Ar dans le Sud Maradi et vers $496 \pm 50$ Ma Rb-Sr dans le NW Nigeria (Ogezi, 1977), jouant ainsi un rôle important dans la circulation des fluides minéralisés (Aboubacar, 2012 ; Garba, 2002 ; Fatiha et al., 2015).

Dans la zone d'étude, la distribution des formations géologiques se traduit par des passages graduels des gneiss à des migmatites (Figure 2). Les schistes forment des bandes d'orientation globalement NE-SW, limitées par des couloirs de cisaillement ("Shear Zone") de même orientation. Ces ensembles sont intrudés par des granitoïdes peu ou pas déformés (Mignon, 1970 ; PRDSM, 2005 ; Baraou, et al., 2018 ; Fati et Konaté, 2020).

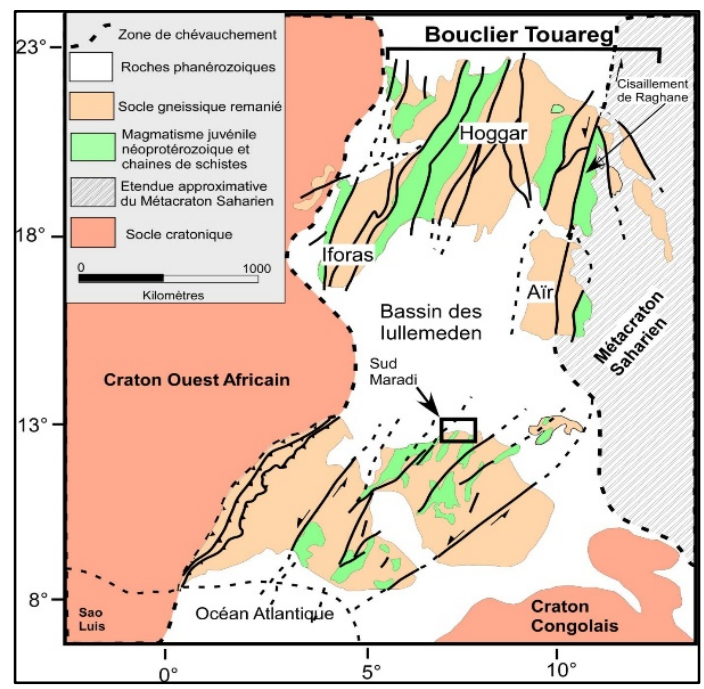

Figure 1.Localisation de la province panafricaine du Sud Maradi dans la chaîne mobile Trans-Saharienne (Castaing et al., 1993 ; Villeneuve and Cornée, 1994; Stern, 1994;

Trompette, 1997; Abdelsalam et al., 2002; Liégeois et al., 2003; Caby, 2003). 


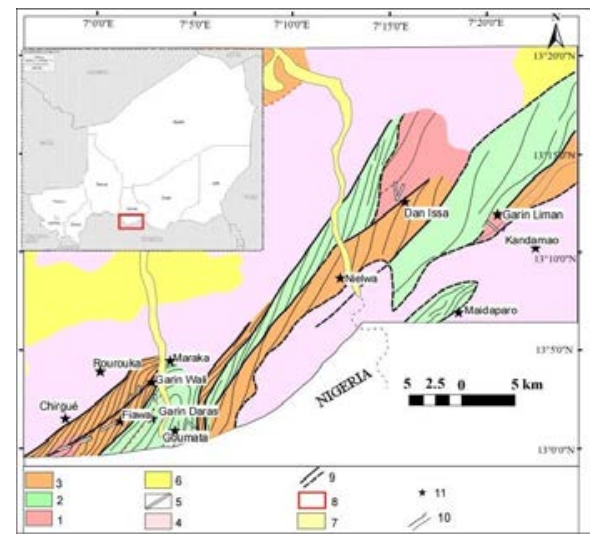

Figure 2. Carte géologique du secteur d'étude compilée à partir des données géophysiques (PRDSM, 2005), cartographiques (Mignon, 1970), de forage et de terrain, montrant la position des coupes réalisées. (1) Gneiss migmatitiques, (2) Ceinture de schistes, (3) Gneiss mylonitiques, (4) Granites peu ou pas déformés, (5) Filons de quartz ou de pegmatite, (6)

Grès du Crétacé, (7) Alluvions, (8) Zone d’étude, (9) Cisaillements panafricains, (10) Trajectoires de la schistosité/foliation, (11) Villages

\section{Matériel et méthodes}

\section{Echantillonnage}

Les échantillonnages ont été réalisés aussi bien dans les roches du socle que dans alluvions "stream sediments" et dans les grès du Crétacé (paléoplacers). Pour les formations de socle, différents faciès pétrographiques (gneiss, granite, quartz et pegmatite) ont été prélevés. Dans les alluvions, des prélèvements ont été opérés à une profondeur de 30 à $40 \mathrm{~cm}$ dans les lits d'oueds (ou koris), drainant les zones de cisaillement affectant le socle. Les grès du Crétacé ont été également échantillonnés à proximité des zones de cisaillement et du socle (figure 3 ).
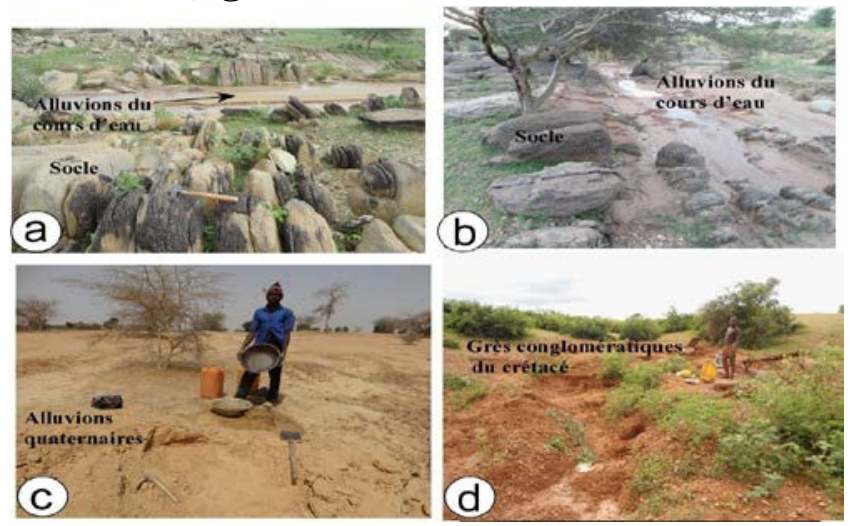

Figure 3 : Sites d'orpaillage dans les alluvions, montrant les différents types d'altérites aurifères, au voisinage des zones de socle. $\mathbf{a}$ et $\mathbf{b}$ : Alluvion de cours d'eaux drainant les éléments du socle, $\mathbf{c}$ : Alluvions quaternaires de la zone du socle, $\mathbf{d}$ : Grès conglomératiques ferrugineux aurifères du Crétacé 


\section{Dosage géochimique}

L'analyse géochimique a consisté à la détermination des teneurs en or par le procédé de la table vibrante (ou table à secousse), au Laboratoire du Génie des Procédés de l'Ecole des Mines de l'Industrie et de la Géologie (EMIG, Niger) et par la méthode du dosage par absorption atomique (Au Fire Assay/AA) au Laboratoire de géochimie ActLabs à Ouaga (Burkina Faso).

Au total, 30 échantillons de socle ont été dosés : soient 11 de gneiss, 7 de schistes, 3 de granites et 9 de roches filoniennes (tableau 1). Parallèlement, 5 échantillons de sédiments ( 2 dans les alluvions, 1 dans le sable quaternaire et 2 dans les grès du Crétacé) ont fait l'objet d'un traitement à la table vibrante (ou table à secousse).

\section{Procédé de la table à secousses}

La table à secousses est employée pour le traitement des particules dont le diamètre varie entre 75 microns et $4,75 \mathrm{~mm}$. L'efficacité varie principalement en fonction de la taille des particules à séparer. Pour une séparation efficace, la différence entre les masses volumiques des particules denses et des particules légères doit être au moins de $1 \mathrm{~g} / \mathrm{cm}^{3}$.

La table à secousses est composée d'une table légèrement inclinée (réglable de $0^{\circ}$ à $6^{\circ}$ ) dans le sens de la largeur et équipée d'un mécanisme qui lui communique longitudinalement un mouvement de va-et-vient asymétrique (Figure 4). L'amplitude et la vitesse de ce mouvement sont réglables. La surface de la table est couverte de rainures longitudinales et parallèles dont la profondeur diminue progressivement à partir du point d'alimentation de la pulpe jusqu’à la sortie, qui est diamétralement opposée (Figure 4). L'effet des secousses entraîne la formation de deux couches de particules distinctes dont l'une est composée de particules de masse volumique élevée (couche inférieure) et l'autre de particules de masse volumique faible (couche supérieure).

Les particules légères (les grains du sable) sont entraînées dans la direction transversale par l'eau de lavage jusqu'au bas de la pente de la table où elles sont collectées. Les particules lourdes sont acheminées longitudinalement jusqu'à l'autre extrémité de la table vibrante grâce à la direction et à la nature asymétrique des secousses.

Pour la méthode à la batée, les teneurs en or dans les alluvions ont été déterminées en faisant le rapport de poids de l'or au poids total de l'échantillon. 


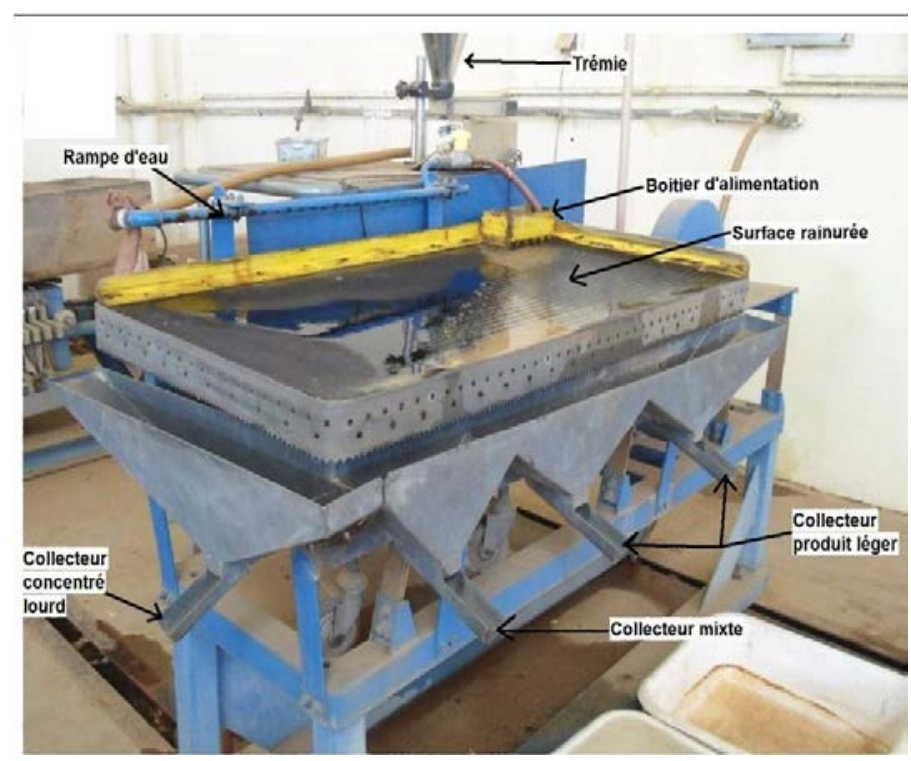

Figure 4. Différentes parties de la table à secousse

\section{Procédé de la méthode d'absorption atomique}

La méthode d'absorption atomique utilisée par le laboratoire ActLabs Burkina Faso est la suivante : prélèvement de 50 ou 30 g représentatif de l'échantillon, préalablement pulvérisé, auquel des fondants basiques sont ajoutés. L'ensemble est ensuite porté à une température de fusion de l'or $\left(1060^{\circ} \mathrm{C}\right)$ dans un four. Après fusion, les métaux sont séparés des scories de silicates pour obtenir un «bouton » au fond du creuset. Ce bouton contient les métaux (y compris l'or). Une fois l'échantillon retiré du four, on procède à la séparation de l'or des autres métaux puis à la concentration de l'or par la spectrométrie d'absorption atomique. (SAA). L'organigramme de la figure 5 illustre le protocole expérimental du dosage de l'or par la méthode de spectrométrie d'absorption atomique (SAA). 


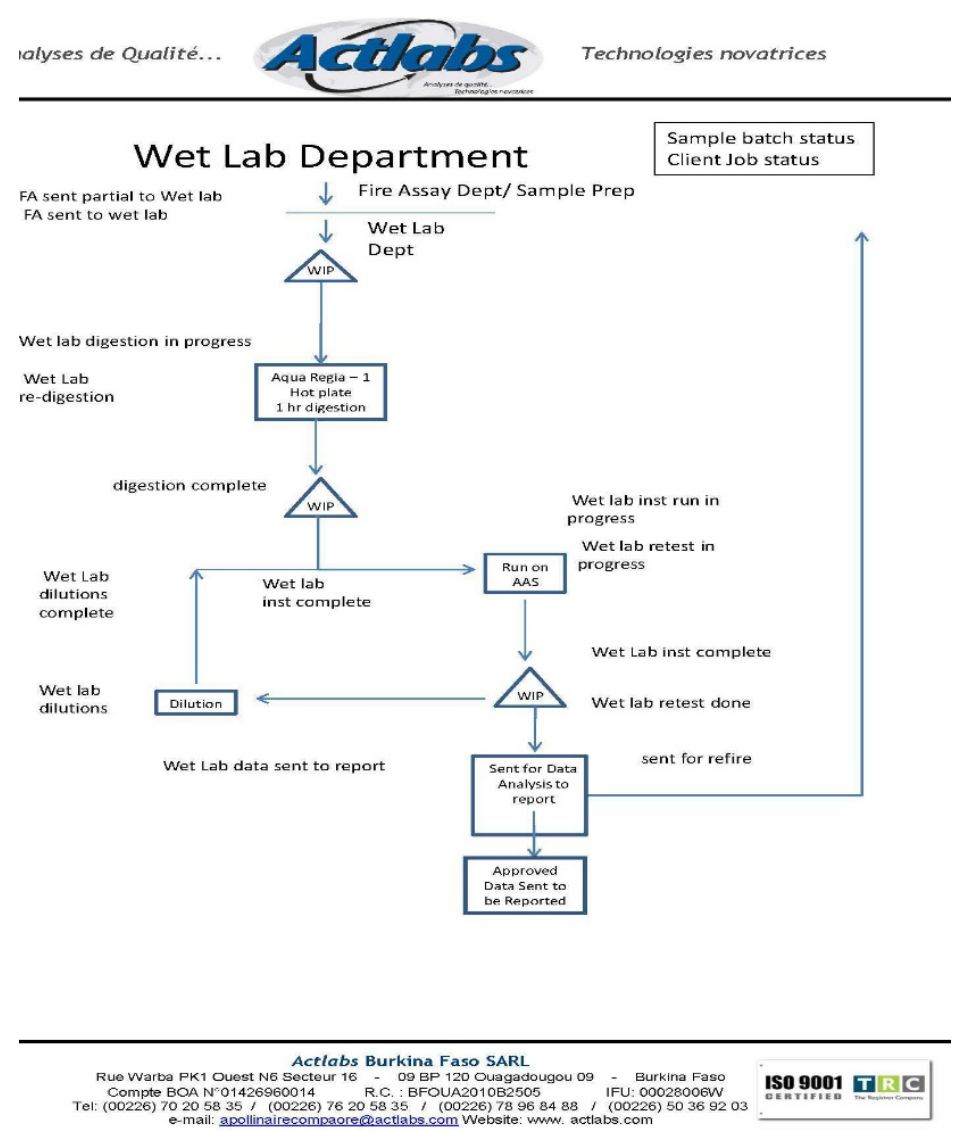

Figure 5. Organigramme du procédé du dosage géochimique de l'or

\section{Résultats et discussions}

\section{Minéralisations aurifères secondaires}

Le dosage de l'or alluvionnaire à la table vibrante a concerné les échantillons prélevés dans les cours d'eau, les grès conglomératiques ferrugineux du Crétacé et les sables quaternaires. Les paillettes d'or obtenues dans les sédiments sont de taille millimétrique (Figure 6). Les échantillons prélevés dans les zones d'orpaillage ont donné de fortes teneurs allant de 5 à $15 \mathrm{~g} / \mathrm{t}$. Ainsi :

- les deux échantillons de grès du Crétacé ont donné des teneurs de 12 $\mathrm{g} / \mathrm{t}$ et de $14 \mathrm{~g} / \mathrm{t}$,

- les teneurs obtenues dans les alluvions varient de 5 à $12 \mathrm{~g} / \mathrm{t}$,

- l'échantillon de sable quaternaire indique une teneur de 15 g/t. 

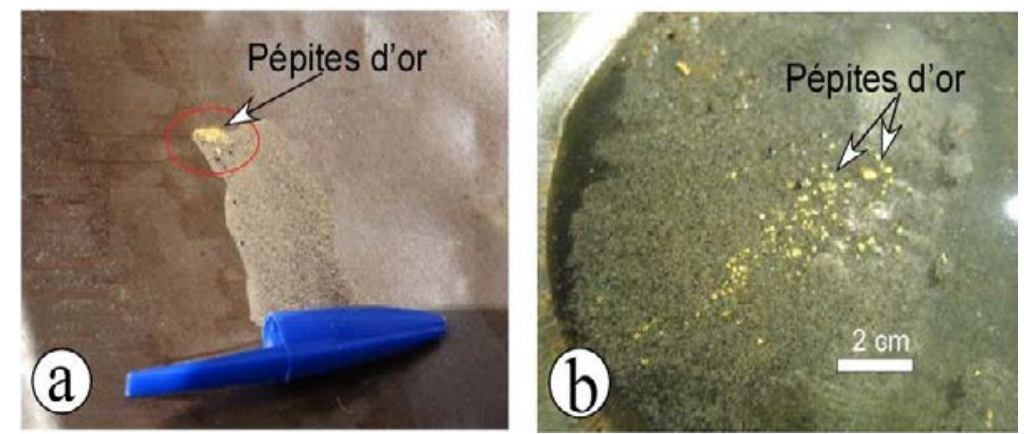

Figure 6. Aspect des paillettes d'or dans les sédiments : en (a) dans le sable quaternaire, en (b) dans le grès conglomératique du Crétacé

Tableau 1. Teneurs en or alluvionnaire obtenues par la table vibrante

\begin{tabular}{|l|l|l|l|l|l|}
\hline $\begin{array}{l}\text { Type de } \\
\text { sédiment } \\
\text { analysé }\end{array}$ & $\begin{array}{l}\text { Teneur } \\
\text { calculée } \\
\text { en or (g/t) }\end{array}$ & $\begin{array}{l}\text { Masse des } \\
\text { échantillo } \\
\text { ns } \\
\text { prélevés } \\
\text { (Kg) }\end{array}$ & $\begin{array}{l}\text { Longitud } \\
\text { e Est (en } \\
\text { degré) }\end{array}$ & $\begin{array}{l}\text { Latitude } \\
\text { Nord } \\
\text { degré) }\end{array}$ & (en \\
\hline $\begin{array}{l}\text { Sable } \\
\text { quaternaire }\end{array}$ & $\mathbf{1 5}$ & 20.50 & 7.04400 & 13.05214 & $\begin{array}{l}\text { Garin } \\
\text { Wali }\end{array}$ \\
\hline $\begin{array}{l}\text { Alluvions de } \\
\text { cours d'eau }\end{array}$ & $\mathbf{1 2}$ & 10.35 & 7.04408 & 13.05375 & $\begin{array}{l}\text { Garin } \\
\text { Wali }\end{array}$ \\
\hline $\begin{array}{l}\text { Alluvions de } \\
\text { cours d'eau }\end{array}$ & $\mathbf{5}$ & 7.6 & 6.98222 & 13.02736 & Chirgué \\
\hline $\begin{array}{l}\text { Grès } \\
\text { conglomératique }\end{array}$ & $\mathbf{1 4}$ & 17.40 & 7.04528 & 13.05447 & $\begin{array}{l}\text { Garin } \\
\text { Wali }\end{array}$ \\
\hline $\begin{array}{l}\text { Grès } \\
\text { conglomératique }\end{array}$ & $\mathbf{1 2}$ & 11.56 & 7.02238 & 13.06244 & Rourouka \\
\hline
\end{tabular}

Afin de mieux visualiser la répartition spatiale des indices aurifères alluvionnaires (placers), les teneurs obtenues par le procédé de la table à secousse ont été complétées par celles des dosages géochimiques multiéléments réalisés par Chalamet (2004) (tableau 2).

Tableau 2.Indices d'or secondaire (alluvionnaire) obtenus par analyse multi-éléments ICP (Chalamet, 2004)

\begin{tabular}{|l|l|l|l|l|l|l|l|l|}
\hline $\begin{array}{l}\text { Teneurs } \\
\text { en Au } \\
(\mathbf{g} / \mathbf{t})\end{array}$ & $\begin{array}{l}\text { Long } \\
(\mathbf{m})\end{array}$ & $\begin{array}{l}\text { Lat } \\
\mathbf{( m )}\end{array}$ & $\begin{array}{l}\text { Teneurs } \\
\text { en Au } \\
\mathbf{( g / t )}\end{array}$ & Long (m) & Lat (m) & $\begin{array}{l}\text { Ten } \\
\text { eurs } \\
\text { en } \\
\text { Au } \\
\mathbf{( g / t )}\end{array}$ & $\begin{array}{l}\text { Long } \\
(\mathbf{m})\end{array}$ & Lat (m) \\
\hline $\mathbf{8}$ & 283100 & $\begin{array}{l}14510 \\
0\end{array}$ & $\mathbf{1 5}$ & 279600 & 1441400 & $\mathbf{1 0}$ & 289150 & 1442750 \\
\hline $\mathbf{5}$ & 284100 & $\begin{array}{l}14403 \\
00\end{array}$ & $\mathbf{1 1}$ & 289400 & 1441500 & $\mathbf{5}$ & 284400 & 1442800 \\
\hline $\mathbf{7}$ & 292200 & 14387 & $\mathbf{8}$ & 292600 & 1441600 & $\mathbf{5}$ & 285250 & 1442800 \\
\hline
\end{tabular}




\begin{tabular}{|l|l|l|l|l|l|l|l|l|}
\hline $\mathbf{6}$ & 282650 & $\begin{array}{l}14387 \\
50\end{array}$ & $\mathbf{8}$ & 294750 & 1441600 & $\mathbf{1 1}$ & 285400 & 1443100 \\
\hline $\mathbf{6}$ & 287900 & $\begin{array}{l}14388 \\
00\end{array}$ & $\mathbf{1 0}$ & 287600 & 1441600 & $\mathbf{5}$ & 293200 & 1443200 \\
\hline $\mathbf{6}$ & 285200 & $\begin{array}{l}14393 \\
00\end{array}$ & $\mathbf{5}$ & 287750 & 1441850 & $\mathbf{8}$ & 284050 & 1443350 \\
\hline $\mathbf{7}$ & 288000 & $\begin{array}{l}14396 \\
00\end{array}$ & $\mathbf{7}$ & 289950 & 1441850 & $\mathbf{6}$ & 285400 & 1443500 \\
\hline $\mathbf{8}$ & 280700 & $\begin{array}{l}14398 \\
00\end{array}$ & $\mathbf{5}$ & 290300 & 1441900 & $\mathbf{1 1}$ & 284900 & 1443600 \\
\hline $\mathbf{5}$ & 288800 & $\begin{array}{l}14403 \\
00\end{array}$ & $\mathbf{9}$ & 283100 & 1442300 & $\mathbf{2 0}$ & 293700 & 1443600 \\
\hline $\mathbf{5}$ & 284750 & $\begin{array}{l}14405 \\
00\end{array}$ & $\mathbf{6}$ & 280900 & 1442400 & $\mathbf{1 2}$ & 285000 & 1444350 \\
\hline $\mathbf{1 0}$ & 288200 & $\begin{array}{l}14405 \\
35\end{array}$ & $\mathbf{6}$ & 282000 & 1442400 & $\mathbf{5}$ & 292350 & 1444400 \\
\hline $\mathbf{5}$ & 285000 & $\begin{array}{l}14408 \\
00\end{array}$ & $\mathbf{2 7}$ & 292650 & 144250 & $\mathbf{1 0}$ & 285640 & 1444450 \\
\hline $\mathbf{5}$ & 284550 & $\begin{array}{l}14409 \\
00\end{array}$ & $\mathbf{1 4}$ & 290050 & 1442500 & $\mathbf{7}$ & 295700 & 1445300 \\
\hline $\mathbf{3 0}$ & 277600 & $\begin{array}{l}14409 \\
50\end{array}$ & $\mathbf{8}$ & 280100 & 1442600 & $\mathbf{6}$ & 286700 & 1445600 \\
\hline $\mathbf{7}$ & 290400 & $\begin{array}{l}14412 \\
00\end{array}$ & $\mathbf{3 3}$ & 288595 & 1442720 & $\mathbf{7}$ & 286800 & 1445900 \\
\hline & & & & & $\mathbf{9}$ & 292500 & 1447200 \\
\hline
\end{tabular}

Les données projetées sur la carte géologique du Sud Maradi (Figure 7) indiquent que les sédiments les plus minéralisés sont situés à proximité des zones de cisaillement du socle ("Shear Zone).

Des investigations ont été également menées sur les altérites du socle. Suivant la lithologie, les teneurs obtenues se répartissent comme suit:

- les schistes présentent des teneurs variant de 5 à $30 \mathrm{~g} / \mathrm{t}$;

- les gneiss mylonitiques indiquent des teneurs allant de 5 à 20 à g/t ;

- tandis que dans les granites les teneurs varient de $8 \mathrm{~g} / \mathrm{t}$ à $15 \mathrm{~g} / \mathrm{t}$.

L’analyse de ces résultats montre ainsi que les éluvions du socle présentent des teneurs en or relativement plus élevées (de 5 à $30 \mathrm{~g} / \mathrm{t}$ ) au même titre que les alluvions (placers) ou les grès du Crétacé (paléoplacers), dépassant largement celles obtenues dans les roches saines du socle (0.006 à $0.017 \mathrm{~g} / \mathrm{t})$. Ce grand écart de concentrations peut s'expliquer par les lessivages importants ayant affecté les roches du socle. Ces lessivages auraient remobilisé, puis reconcentré l'or dans les sédiments. Des observations similaires ont été réalisées au Nord-Ouest du Nigéria, dans le secteur de Maru-Anka, où la zone d'altération longeant les zones de cisaillement présente de fortes concentrations en or (Talaat et Mohammed, 2010). 


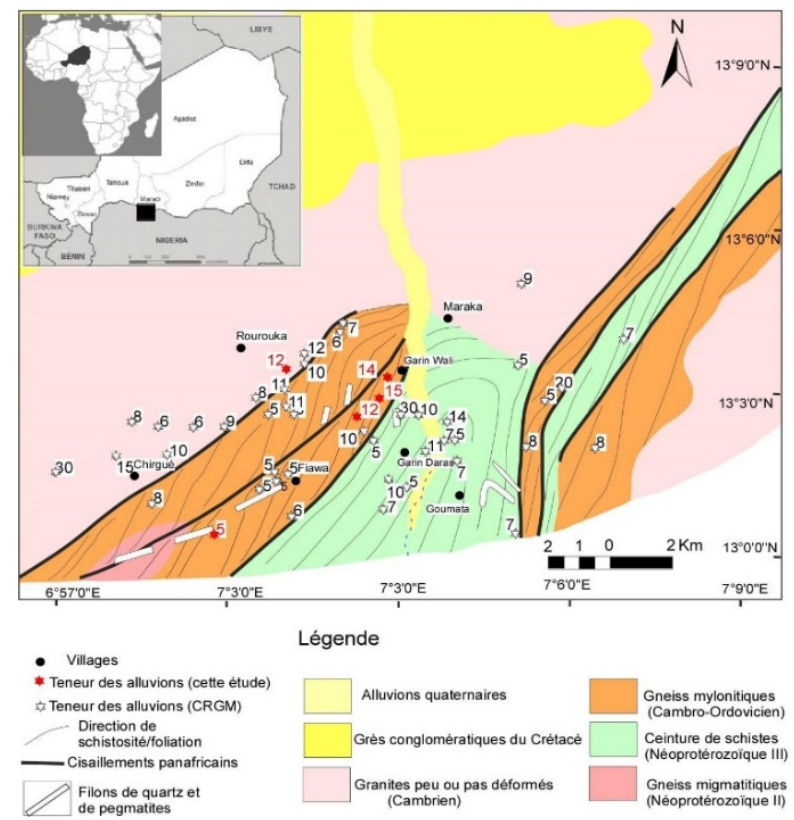

Figure 7.Carte géologique de la zone d'étude montrant la localisation et les teneurs des échantillons alluvionnaires en gramme par tonne. Les étoiles rouges représentent les teneurs obtenues au cours de cette étude et les étoiles blanches celles provenant de Chalamet (2004)

\section{Indices d'or dans les formations de socle (indices primaires)}

Les teneurs en or sont exprimées en ppm ou en g/t. Sur les trente (30) échantillons dosés, seize (15) présentent des teneurs $<0.005$ g/t (c’est la limite de détection du laboratoire ACTLAbs de Ouagadougou). Les teneurs supérieures à $0.005 \mathrm{~g} / \mathrm{t}$ sont projetées sur la carte géologique de la zone d'étude (Figure 8). Les teneurs obtenues par le dosage de l'or par absorption atomique sont consignés dans le tableau 3 et se répartissent comme suit :

Les gneiss mylonitiques présentent des teneurs variant de 0.006 à $0.017 \mathrm{~g} / \mathrm{t}$,

Les schistes indiquent des teneurs variant de 0.006 à $0.013 \mathrm{~g} / \mathrm{t}$,

Dans les granites post-tectoniques les teneurs sont $\leq 0.006 \mathrm{~g} / \mathrm{t}$. Il en est de même pour les filons de quartz et de pegmatite $(\leq 0.006 \mathrm{~g} / \mathrm{t})$.

Ces résultats indiquent que les minéralisations primaires de l'or sont à l'état disséminé dans les formations du socle (faibles teneurs de 0.005 à 0.017 $\mathrm{g} / \mathrm{t}$ ) et ne semblent pas être liées à une formation géologique particulière.

La teneur de coupure permettant la séparation entre la minéralisation et l'encaissant varie selon les exploitations minières et en fonction du contexte géologique. Elle est de l'ordre de 0,3 g/t pour la mine de Samira au Niger, 0,74 $\mathrm{g} / \mathrm{t}$ à Siguiri en Guinée (Lebrun et al., 2017) 0,60 g/t à Morila au Mali et 0,98 à Bamboré au Burkina Faso (Robertson and Peters, 2016). Comparées à ces teneurs de coupure, les valeurs obtenues dans le Sud Maradi, variant de 0.005 
à $0.017 \mathrm{~g} / \mathrm{t}$, indiquent que toutes les formations de socle sont très faiblement minéralisées.

Tableau 3. Résultats du dosage par absorption atomique des formations de socle exprimé en gramme par tonne $(\mathrm{g} / \mathrm{t})$. Les teneurs considérées comme étant positives sont représentées en

\begin{tabular}{|l|l|l|l|l|}
\hline Echantillons & $\begin{array}{l}\text { Teneur en } \\
\text { Au (g/t) }\end{array}$ & $\begin{array}{l}\text { Longitude } \\
\text { Est (en } \\
\text { degré) }\end{array}$ & $\begin{array}{l}\text { Latitude } \\
\text { Nord (en } \\
\text { degré) }\end{array}$ & Localité \\
\hline Filon d'aplite & $<0.005$ & 7.0750270 & 13.018861 & Goumata \\
\hline Filon d'aplite & $<0.005$ & 7.0495000 & 13.049500 & Kwari \\
\hline Filon pegmatite & $<0.005$ & 6.9822200 & 13.027360 & Chirgué \\
\hline Filon pegmatite & $\mathbf{0 . 0 0 6}$ & 7.0353000 & 13.049700 & Dan ali \\
\hline Filon pegmatite & $\mathbf{0 . 0 0 6}$ & 7.0284444 & 13.042388 & Fiawa \\
\hline Filon pegmatite & $<0.005$ & 7.0440800 & 13.053750 & Garin Wali \\
\hline Filon quartz blanc & $<0.005$ & 7.0179000 & 13.022700 & Fiawa \\
\hline Filon quartz gris & $\mathbf{0 . 0 0 5}$ & 7.0490000 & 13.033000 & Garin Darras \\
\hline Filon quartz gris & $<0.005$ & 7.0779440 & 13.021972 & Goumata \\
\hline Gneiss à amphibole & $\mathbf{0 . 0 0 6}$ & 7.092167 & 13.021806 & Goumata \\
\hline Gneiss mylonitique & $\mathbf{0 . 0 0 6}$ & 7.046814 & 13.052839 & Kwari \\
biotite & & & & \\
\hline Gneiss migmatitique 1 & $<0.005$ & 6.9736110 & 13.005694 & Chirgué \\
\hline Gneiss migmatitique 2 & $\mathbf{0 . 0 0 9}$ & 6.9744450 & 13.004440 & Chirgué \\
\hline Gneiss Migmatitique 3 & $\mathbf{0 . 0 0 6}$ & 7.3366110 & 13.192500 & Garin Liman \\
\hline Gneiss Migmatitique 4 & $<0.005$ & 7.3366110 & 13.192500 & Garin Liman \\
\hline Gneiss mylonitique 1 & $\mathbf{0 . 0 1 7}$ & 7.2659440 & 13.191055 & Dan Issa \\
\hline Gneiss mylonitique 2 & $<0.005$ & 7.0256670 & 13.021306 & Fiawa \\
\hline Gneiss mylonitique 3 & $<0.005$ & 7.0473610 & 13.045138 & Garin Kwari \\
\hline Gneiss mylonitique 4 & $\mathbf{0 . 0 0 9}$ & 7.0422220 & 13.053277 & Garin Wali \\
\hline Gneiss mylonitique 5 & $\mathbf{0 . 0 1 7}$ & 7.0879170 & 13.022667 & Goumata \\
\hline Granite 1 & $<0.005$ & 7.0234000 & 13.040600 & Dan Ali \\
\hline Granite 2 & $<0.005$ & 7.0051940 & 13.025750 & Fiawa \\
\hline Granite 3 & $\mathbf{0 . 0 0 6}$ & 7.0284440 & 13.042389 & Rourouka \\
\hline Micaschiste 1 & $<0.005$ & 7.046416 & 13.00622 & Doufoumara \\
\hline Micaschiste 2 & $\mathbf{0 . 0 0 6}$ & 7.0464160 & 13.006220 & Doufoumara \\
\hline Micaschiste 3 & $\mathbf{0 . 0 0 6}$ & 7.0464160 & 13.006220 & Doufoumara \\
\hline Micaschiste 4 & $<0.005$ & 7.0414720 & 13.023611 & Karamné \\
\hline Schiste 1 & $\mathbf{0 . 0 1 3}$ & 7.0369170 & 13.020833 & Fiawa \\
\hline schiste 2 & $\mathbf{0 . 0 0 9}$ & 7.0490000 & 13.033000 & Garin Darras \\
\hline Schiste 3 & $<0.005$ & 7.0503610 & 13.023111 & Karamné \\
\hline
\end{tabular}

\section{Interprétation des données}

L’analyse des résultats du dosage géochimique des roches du socle indique que les minéralisations primaires de l'or sont à l'état disséminé (faibles teneurs de 0.005 à $0.017 \mathrm{~g} / \mathrm{t}$ ). Au contraire, au voisinage des zones de cisaillement (Figure 7) les teneurs enregistrées sont relativement plus 
importantes aussi bien dans les formations de socle $(0.006$ à $0.017 \mathrm{~g} / \mathrm{t})$ que dans les sédiments (5 à $30 \mathrm{~g} / \mathrm{t}$ ). Il ressort de ces résultats que les teneurs en or dans les sédiments sont bien plus élevées que celles enregistrées dans les formations de socle.

Dans la province contiguë du Nord Nigéria, les études réalisées ont montré également que les minéralisations aurifères sont spatialement associées aux zones de cisaillement (Talaat et Mohammed, 2010). Par exemple, la zone de cisaillement d'Anka, Zuru (au Nord-Ouest Nigéria) représente un important couloir prospectif qui s'étend jusqu'au Niger, dans le Sud Maradi (Garba, 2000, 2002, Talaat et Mohammed, 2010, Baraou, 2018). Au Nord-Ouest du Nigéria, dans les zones de cisaillement altérées les teneurs en or peuvent atteindre $39 \mathrm{~g} / \mathrm{t}$ (Talaat et Mohammed, 2010). En Guinée également, dans certaines zones de faille, les teneurs peuvent dépasser $100 \mathrm{~g} / \mathrm{t}$ (Lebrun et al., 2017). Le fait que les indices aurifères primaires soient relativement plus élevés dans les "Shear Zones" orientées $\sim \mathrm{N} 40^{\circ}$ (Baraou et al., 2018, Baraou et Konaté, 2020), ou dans leur voisinage, suggèrent qu'une activité thermo-tectonique tardi à post-panafricaine aurait exercé un rôle important dans les "remobilisations" de l'or. Ces zones de cisaillement représenteraient des conduits favorables au mouvement de fluides minéralisateurs (Abubakar, 2012), tandis que les schistes graphiteux constituent une barrière chimique pour la précipitation de la minéralisation de sulfure d'or (Garba, 2002). Ce type de contrôle tectonique dans la minéralisation en or a été observé dans le gisement d'or d'Ashanti Gold au Ghana (Mumin et al., 1994).

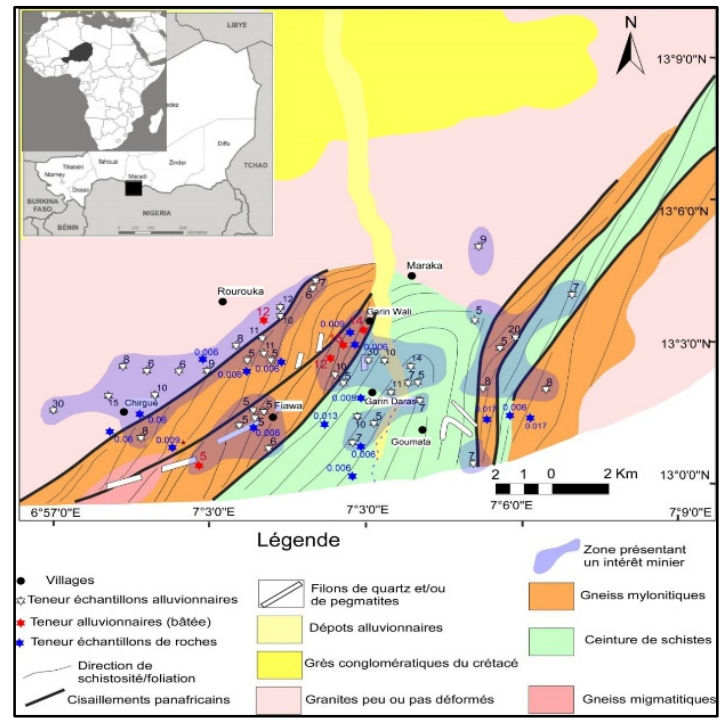

Figure 8. Carte géologique du secteur d'anomalie du Sud Maradi montrant la localisation et teneurs des échantillons du socle et des alluvions (D’après Mignon, 1970; modifiée et complétée) 


\section{Modèle de distribution des indices aurifères du Sud Maradi}

La distribution de l'or dans les formations du Sud Maradi pourrait se faire en deux étapes :

1. La première correspondrait à une dissémination de la minéralisation dans les roches de la ceinture et dans les couloirs de cisaillements tardipanafricains d'orientation NE-SO ("or invisible"), représentées par des schistes et des gneiss mylonitiques ou rubanés.

2. La seconde correspondrait à la formation de paillettes d'or de taille infra millimétrique dans les placers récents (alluvions et sables quaternaires) à millimétrique dans les paléoplacers (grès conglomératiques du Crétacé), comme résultant d'une remobilisation postérieure de l'or formé au cours de la première étape, "or visible" (concept emprunté à Sangaré et al., 2014). Une telle configuration a été décrite dans certains gisements birimiens du Craton Ouest Africain, dont Bogoso-Prestea au Ghana (Mumin et al., 1994) et Sabodala au Sénégal (Sylla et al., 1997), en Côte d'Ivoire dans le gisement de Dougbafla (Ouattara et al., 2017, Ouattara et al., 2016 ). Dans le cas du gisement ghanéen (Bogoso-Prestea), la minéralisation tardive à "or visible" serait le produit d'une remobilisation de l'"or invisible" (Mumin et al., 1994) dans les grains d'arsénopyrite (Mumin et al., 1994). Ce qui n'est pas le cas dans le Sud Maradi, où les paillettes d'or alluvionnaires et des sédiments ne sont pas associées à des minéraux. Dans le cas du gisement sénégalais (Sabodala), la remobilisation se produit à la suite d'une déformation cassante postérieure au cisaillement ductile (Sylla et al., 1997). Un tel scénario pourrait expliquer les fortes teneurs de coupure observées dans les minéralisations secondaires observées dans le Sud Maradi (5 à 30 g/t).

Dans le drainage en aval les zones de cisaillement, à minéralisation aurifère primaire, une quantité importante d'or pourrait provenir de l'altération météorique (Figure 9). L’or invisible capté par le système de drainage (Figure 9), pourrait alors soit précipiter soit former des agrégats à une certaine distance en aval de la zone minéralisée ; ce qui aurait conduit dans le Sud Maradi à des teneurs économiquement significatives.

Par ailleurs, la présence de paillettes d'or dans les alluvions de la zone étudiée (secteur de Garin Wali, Figure 6) pourrait s'expliquer par des lessivages lointains des formations de socle du Nord-Ouest du Nigéria. En effet, dans les zones de cisaillement de Garin Hawal, Birnin-Yauri-Zuru, Anka (Nord-Ouest Nigéria), Talaat et Mohammed (2010) ont mis en évidence des larges zones d'altération présentant des forts indices d'or or (0.3 à $10 \mathrm{~g} / \mathrm{t}$ ). Dans ces zones d'altération, "l’or visible" est associé aux placers à éléments d'amphibolites. 


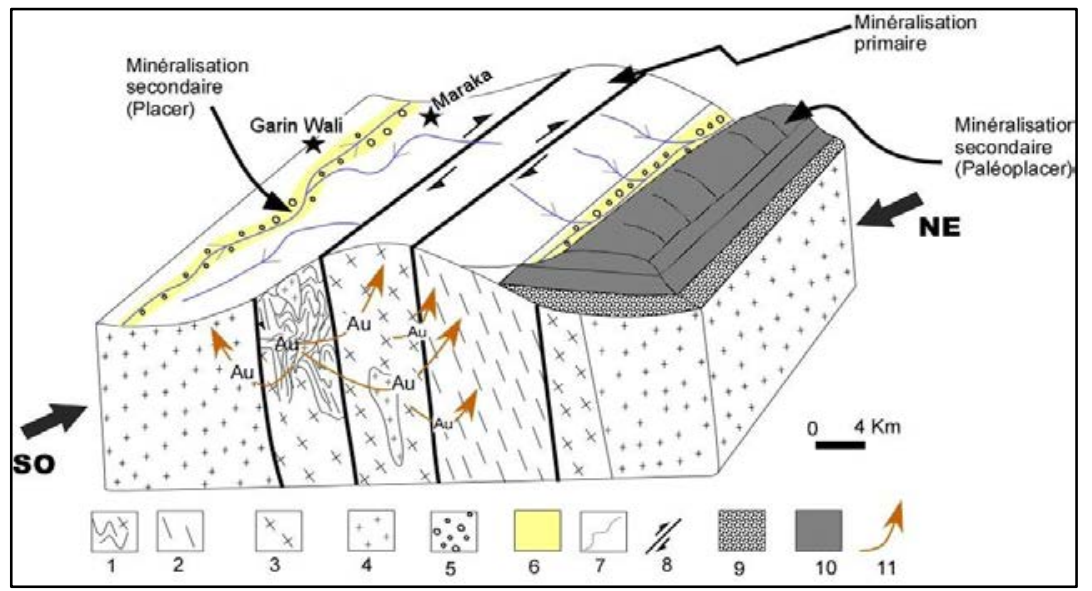

Figure 9. Modèle de la minéralisation aurifère du Sud Maradi. Ce modèle part de l'hypothèse selon laquelle : la collision continent-continent conduit au métamorphisme des schistes et des plutons granitoïdiques, d'où une fusion partielle localisée favorisant la minéralisation primaire de l'or dans les couloirs de cisaillement. Le lessivage du complexe métamorphique conduit à une remobilisation et à une minéralisation secondaire. 1. Gneiss migmatitiques, 2 : Ceinture de schistes, 3 : Gneiss mylonitiques, 4 : Granites peu ou pas déformés, 5 : Grès du Crétacé, 6 : alluvions récentes (placer), 7 : Cours d’eau, 8 :

Cisaillements panafricains dextres, $\mathbf{9}:$ Grès du Crétacé, $\mathbf{1 0}:$ sables quartenaires (paléoplacer), 11 : sens probable de dissémination de l'or

\section{Conclusion}

Il ressort de ces résultats que les teneurs en or dans les sédiments sont bien plus élevées que celles enregistrées dans les formations de socle. Ces résultats indiquent que les minéralisations primaires de l'or sont à l'état disséminé dans les formations du socle (faibles teneurs de 0.005 à $0.017 \mathrm{~g} / \mathrm{t}$ ) et ne semblent pas être liées à une formation géologique particulière. Les sédiments les plus minéralisés sont situées à proximité des zones de cisaillement du socle ("Shear Zone). La distribution de l'or dans les formations du Sud Maradi pourrait se faire en deux étapes : La première correspondrait à une dissémination de la minéralisation primaire dans les schistes et dans les couloirs de cisaillements tardi-panafricains d'orientation NE-SO. La seconde correspondrait à l'altération supergène des zones de cisaillement du socle ayant favorisé une redistribution des teneurs en or conduisant à des concentrations secondaires plus importantes dont l'exploitation éventuelle ne pourra se faire que par lixiviation.

En perspective, des études sur les inclusions de fluides et les éléments traceurs comme le $\mathrm{Cu}$, le $\mathrm{Pb}$, Sb ou As pour permettre un bon marquage des roches porteuses de la minéralisation. 


\section{References:}

1. Abdelsalam, M. G., Liegeois, J. P. and Stern R. J. (2002). The Saharan Metacraton. Journal of African Earth Sciences, vol. 34, no. 3-4, pp. 119-136.

2. Abubakar, Y. I. (2012). An Integrated Technique in Delineating Structures: A Case Study of the Kushaka Schist Belt Northwestern Nigeria. International Journal of Applied Science and Technology, Vol. 2 No. 5; May 2012, pp. 164-173.

3. Baraou, I.S., Konaté M., Ahmed, Y., Abdoul, W. D. (2018). Caractérisation de la déformation du socle de la zone mobile panafricaine du Sud Maradi, Sud Niger. Afrique Science 14(1), 156170.

4. Baraou, S. I. and Konaté. M. (2020). Kinematic evolution of the PanAfrican shear zones of South Maradi, southern Niger. Glo. J. Earth Environ. Sci, Volume 5(4), pages 101-116. https://doi.org/10.31248/GJEES2020.087

5. Caby, R. (2003). Terrane assembly and geodynamic evolution of Central-Western Hoggar: a synthesis. Journal of African Earth Sciences, 37, 133-159.

6. Castaing, C., Triboulet, C., Feybesse J.L. and Chèvremont, P. (1993). Tectonometamorphic evolution Ghana, Togo and Benin in the light of Pan-African /Bresikiano orogeny. Tectnophysis, 218, 323-342.

7. Chalamet, D. (2004). Rapport de mission de terrain dans le sud Maradi (CRGM). p.35-63.

8. Dada, S.S. (1998). Crust-forming ages and proterozoic crustalevolution in Nigeria: a reappraisal of current interpretations. Precambrian Research 87, 65-74.

9. Fati, B. et konaté, M. (2020). Utilisation de la télédétection et du SIG pour la cartographie des zones minéralisées en or dans la province panafricaine du Sud est Niger (Sud Maradi et Damagaram-Mounio). Rev. Ivoir. Sci. Technol., 36 (2020) 230 - 252.

10. Fatiha, H., Ahmed, A., Abdellah, A. (2015). Etude sédimentologique du Trias et Jurassique du Haut Atlas de Marrakech, Maroc : contribution à la localisation des niveaux favorables à l'exploitation artisanale pour la poterie. European Scientific Journal, vol.11, No.21, 308-333.

11. Fitches, W. R., Ajibade, A. C., Egbuniwe, I. G., Holt, R. W., \& Wright, J. B. (1985). Late Proterozoic schist belts and plutonism in NW Nigeria. Journal of the Geological Society, London, vol. 142, no. 2, pp. 319-337.

12. Grant, N. K. (1970). Geochronology of Precambrian basement rocks from Ibadan, southwestern Nigeria. Earth Planet. Sci. Lett. 10:29-38. 
13. Garba, I. (2002). Geochemical characteristics of the gold mineralization near Tsohon Birnin Gwari, northwestern Nigeria. Chemie der Erde, vol. 62, no. 2, pp. 160-170.

14. Garba, I. (2000). Origin of Pan-African mesothermal gold mineralisation at Bin Yauri, Nigeria. Journ African Earth Sci 31, 433449.

15. Goh, D. (2016). L'exploitation Artisanale De L'or En Côte D'Ivoire: La Persistance D'une Activité Illegale. European Scientific Journal, vol.12, No.3, 18-36.

16. doi: 10.19044/esj.2016.v12n3p18.

17. KENNEDY, W. Q. (1964). The structural Differentiation of Africa in The Pan-African (500 m. y.) Tectonic Episode. Res. Inst. Afr. Geol., 8th Ann. rep., Univ. LeEds, 48-49.

18. Liégeois, J. P., Latouche, L., Boughrara M., Navez, J. and Guiraud, M. (2003). The LATEA metacraton. (Central Hoggar, Tuareg shield, Algeria): behaviour of an old passive margin during the Pan-African orogeny. Journal of African Earth Sciences, 37 (2003), 161-190 p.

19. Lebrun, E., Miller, J., Thébaud, N., Ulrich S. and Campbell, McCuaig T. (2017). Structural Controls on an Orogenic Gold System: The World-Class Siguiri Gold District, Siguiri Basin, Guinea, West Africa. Society of Economic Geologists, Inc., Economic Geology, v. 112, pp. 73-98.

20. Mignon, R. (1970). Etude géologique et prospection du Damagaram Mounio et du Sud Maradi, 46 - 51 p. et 54.

21. Mumin, A.H., Fleet M.E. and Chyssoulis, S.L. (1994). Gold mineralization in As-rich mesothermal gold ores of Bogosu-Presta mining district of the Ashanti Gold Belt, Ghana: remobilization of "invisible" gold. Mineralum Deposita, 29, 445-460.

22. Ouattara, A. S., Coulibaly, Y., Kouadio, Fossou, J-L. H. (2017). Les Altérations Hydrothermales Associées à la Minéralisation Aurifère Du Gisement De Dougbafla (District d'Oumé-Hiré, Centre-Ouest De La Côte d'Ivoire). European Scientific Journal, Vol.13, No.30, 108-125. Doi: 10.19044/esj.2017.v13n30p108.

23. Ouattara, A. S., Baka, D., Ouattara, G., Nimaga, A. (2016). Mise En Evidence De Nouvelles Cibles De Forages à Partir De L'analyse De La Fracturation Du Prospect Aurifère De Dougbafla-OUME (CentreOuest De La Côte d'Ivoire). European Scientific Journal, vol.12, No.36, 336-354. doi: 10.19044/esj.2016.v12n36p336.

24. PRDSM. (2005). Projet de géophysique aéroportée dans le secteur Sud Maradi. Ministère des Mines et de développement Industriel, 55 p.

25. Robertson, M. And Peters, L. (2016). West African Goldfields. Episodes Vol. 39, no. 2, pp 155-176. 
26. Samuel, O. A., and Fakorede, O. (1988). Gold Mineralization in the Nigerian schist Belts. Bicentennal Gold 88, Melbourne, May, 1988, pp14-142.

27. Stern, R. J. (1994). Arc assembly and continental collision in the Neoproterozoic East African orogeny - implications for the consolidation of Gondwana. Annual Review of Earth and Planetary Sciences, Vol. 22, pp. 319-351.

28. Sylla, M., Ngom, P. M. (1997). Le gisement d’or de Sabodala (Sénégal oriental): une minéralisation filonienne d'origine hydrothermale remobilisée par une tectonique cisaillante. J. Afr. Earth Sci., 25 (2), 183-192

29. Sangaré, A., Driouch, Y., Salvi S., Féménias, O., Siebenaller, L., Belkasmi M., Béziat, D., Dahire, M., Ntarmouchan,T A., Adil, S. and Debat, P. (2014). Géologie des minéralisations aurifères du gisement tardi-éburnéen de Kalana (Birimien, Sud-Ouest du Mali). Bulletin de l'Institut Scientifique, Rabat, Section Sciences de la Terre, 2014, $\mathrm{n}^{\circ}$ 36, 00 - 00.

30. Talaat, M. R., and Mohammed, F. A. F. (2010). Characterization of gold mineralization in Garin Hawal area, Kebbi State, NW Nigeria, using remote sensing. The Egyptian Journal of Remote Sensing and Space Sciences (2010) 13, 153-163.

31. Turner, D. C. (1983). "Upper Proterozoic Schist Belts in the Nigerian Sector of the Pan-African Province of West-Africa", Precambrian Research, vol. 21, no. 1-2, pp. 55-79.

32. Trompette, R. (1997). Neoproterozoic ( 600 Ma) aggregation of Western Gondwana: a tentative scenario. Precambrian Research, vol. 82, pp. 101-112.

33. Villeneuve, M. and Cornée, J. J. (1994). Structure, evolution and palaeogeography of West African craton and bordering belts during the Neoproterozoic. Prec. Res., 69, 307-326.

34. Wright, J. B., Hastings, D.A., Jones W.B., Williams H.R. (1985) Geology and mineral resources of West Africa. Allen \& Unwin, London, 187 p. 